\title{
Disparities in Risk Perception of Thyroid Cancer Recurrence and Death
}

\author{
Debbie W. Chen, MD (DD '; David Reyes-Gastelum, MSc'; Lauren P. Wallner, PhD, MPH (iD) 2; Maria Papaleontiou, MD'; \\ Ann S. Hamilton, $\mathrm{PhD}^{3}$; Kevin C. Ward, PhD ${ }^{4}$; Sarah T. Hawley, PhD, MPH²; Brian J. Zikmund-Fisher, PhD ${ }^{5,6}$; \\ and Megan R. Haymart, MD iD 1
}

\begin{abstract}
BACKGROUND: To the authors' knowledge, studies regarding risk perception among survivors of thyroid cancer are scarce. METHODS: The authors surveyed patients who were diagnosed with differentiated thyroid cancer from the Surveillance, Epidemiology, and End Results registries of Georgia and Los Angeles County (2632 patients; $63 \%$ response rate). The analytic cohort was defined by a $\leq 5 \%$ risk of disease recurrence and mortality (1597 patients). Patients estimated their recurrence and mortality risks separately (increments of $10 \%$ and endpoints of $\leq 5 \%$ and $\geq 95 \%$ ). Both outcomes were dichotomized between reasonably accurate estimates (risk perception of $\leq 5 \%$ or $10 \%$ ) versus overestimation (risk perception of $\geq 20 \%$ ). Multivariable logistic regression was used to identify factors associated with risk overestimation, and the relationships between overestimation and both worry and quality of life were evaluated. RESULTS: In the current study sample, $24.7 \%$ of patients overestimated their recurrence risk and $12.5 \%$ overestimated their mortality risk. A lower educational level was associated with overestimating disease recurrence ( $\leq$ high school diploma: odds ratio [OR], 1.64 [95\% Cl, 1.16-2.31]; and some college: OR, 1.36 [95\% Cl, 1.02-1.81]) and mortality ( $\leq$ high school diploma: OR, 1.86 [95\% Cl, 1.18-2.93]) risk compared with those attaining at least a college degree. Hispanic ethnicity was found to be associated with overestimating recurrence risk (OR, 1.44, 95\% Cl 1.02-2.03) compared with their white counterparts. Worry about recurrence and death was found to be greater among patients who overestimated versus those who had a reasonably accurate estimate of their risk of disease recurrence and mortality, respectively $(P<.001)$. Patients who overestimated mortality risk also reported a decreased physical quality of life (mean T score, 43.1; 95\% Cl, 41.6-44.7) compared with the general population. CONCLUSIONS: Less educated patients and Hispanic patients were more likely to report inaccurate risk perceptions, which were associated with worry and a decreased quality of life. Cancer 2020;126:1512-1521. () 2019 American Cancer Society.
\end{abstract}

KEYWORDS: health care disparities, Hispanics, mortality, quality of life, recurrence, thyroid cancer.

\section{INTRODUCTION}

The majority of patients with differentiated thyroid cancer (DTC) have low-risk disease. ${ }^{1-4}$ Despite having an excellent prognosis, prior work has shown that patient worry regarding disease recurrence and death is common, with underrepresented minority groups especially at risk. ${ }^{5}$ In addition, there are conflicting data concerning patient quality of life (QOL), with some studies finding that patients with thyroid cancer have a worse QOL compared with those with melanoma, colorectal cancer, and the general population, and other studies reporting that their QOL is similar to that of healthy patients without cancer. ${ }^{6-9}$ To the best of our knowledge, the reasons for the discordance between patient prognosis and patient worry and QOL remain unknown.

Although studies regarding disease recurrence and mortality risk perception among patients with thyroid cancer are lacking, it is plausible that inaccurate risk estimates by patients might contribute to persistent cancer-related worry and poor QOL. In particular, to our knowledge, there is little information regarding which survivors of thyroid cancer with a favorable prognosis are at risk of overestimating their risk of recurrence and death. The identification of survivors of thyroid cancer who are vulnerable to inaccurate risk perceptions is needed to effectively tailor risk communication and patient education.

The goal of the current study was to determine the level of perceived risk of disease recurrence and of mortality from thyroid cancer among low-risk patients. We hypothesized that overestimation of recurrence and mortality risk would be

\footnotetext{
Corresponding Author: Megan R. Haymart, MD, Division of Metabolism, Endocrinology, and Diabetes, Michigan Medicine, North Campus Research Complex, 2800 Plymouth Rd, Building 16, Room 408E, Ann Arbor, MI 48109 (meganhay@med.umich.edu).

${ }^{1}$ Division of Metabolism, Endocrinology, and Diabetes, University of Michigan, Ann Arbor, Michigan; ${ }^{2}$ Department of Internal Medicine, University of Michigan, Ann Arbor, Michigan; ${ }^{3}$ Department of Preventive Medicine, University of Southern California, Los Angeles, California; ${ }^{4}$ Department of Epidemiology, Emory University, Atlanta, Georgia; ${ }^{5}$ Department of Health Behavior and Health Education, University of Michigan, Ann Arbor, Michigan; ${ }^{6}$ Division of General Internal Medicine, University of Michigan, Ann Arbor, Michigan
}

Brittany Gay assisted with the table and figures.

Additional supporting information may be found in the online version of this article.

DOI: 10.1002/cncr.32670, Received: October 14, 2019; Revised: November 17, 2019; Accepted: November 26, 2019, Published online December 23, 2019 in Wiley Online Library (wileyonlinelibrary.com) 
associated with both increased cancer-related worry and decreased QOL.

\section{MATERIALS AND METHODS}

\section{Study Population and Data Collection}

Between February 1, 2017, and October 31, 2018, we conducted a large, cross-sectional, population-based survey of patients aged 18 to 79 years who were diagnosed with DTC between January 1, 2014, and December 31, 2015. Patients were sampled from the Surveillance, Epidemiology, and End Results (SEER) registries of Georgia and Los Angeles County. To improve response rates, a modified Dillman method was used, which consisted of a $\$ 20$ cash incentive included with the initial mailing, contact tracing, follow-up telephone calls, and multiple mailings to nonresponders. ${ }^{10}$ Patients with Spanish surnames were mailed surveys in both English and Spanish, and bilingual interviewers conducted followup telephone calls. Survey data were entered electronically using a double entry method to ensure $<1 \%$ error. Survey responses were merged with clinical cancer data from the respective SEER registries to create a deidentified data set. The study was approved by the institutional review boards of the University of Michigan, the University of Southern California, Emory University, the California Committee for the Protection of Human Subjects, the California Cancer Registry, and the Georgia Department of Public Health.

Of the 4317 patients identified and mailed a survey, a total of 4185 were determined to be eligible. A total of 2632 patients responded, yielding a 63\% response rate, calculated as the number of respondents divided by eligible patients, and a 77\% cooperation rate, calculated as the number of respondents divided by all patients who were able to be contacted. ${ }^{11}$ For these analyses, we restricted the analytic sample to low-risk patients with both a clinically determined $\leq 5 \%$ risk of persistent structural or recurrent thyroid cancer and a 10-year mortality risk $\leq 5 \%$ (1597 patients) (Fig. 1). All patients in the analytic sample had intrathyroidal classic papillary or follicular thyroid carcinoma with no evidence of lymph node or distant metastases.

To determine patients' clinical 10-year risk of persistent structural or recurrent thyroid cancer, we adapted information from the 2015 American Thyroid Association risk stratification system and findings by Tuttle et al regarding recurrence risk estimates in patients with DTC. ${ }^{1,12}$ We defined such risk to be $\leq 5 \%$ in patients with an intrathyroidal papillary or follicular thyroid cancer measuring $\leq 4 \mathrm{~cm}$ with no evidence of lymph node or distant metastases. We excluded patients whose risk of persistent structural or recurrent thyroid cancer was $>5 \%$ (992 patients) or unknown (43 patients). This exclusion category included patients with more aggressive variants of papillary or follicular thyroid cancers because their risk of disease recurrence would be higher. These aggressive variants were identified using the following SEER International Classification of Diseases for Oncology, Third Edition (ICD-O-3) morphology codes for thyroid cancer diagnosis: 8290 (Hurthle cell cancer), 8332 (trabecular follicular thyroid cancer), 8337 (insular cancer), and 8344 (columnar cell variant of papillary thyroid cancer).

Patients' clinical 10-year mortality risk was determined using age at the time of diagnosis and SEER Summary Stage to assign individuals to 1 of 4 distinct prognostic groups identified by Banerjee et al in their study of 43,392 patients with well-differentiated thyroid cancer using SEER data. ${ }^{2,13}$ Mortality risk was defined as $\leq 5 \%$ in patients of any age with localized disease or those aged $\leq 63$ years with regional disease.

\section{Measures}

The survey instrument was developed based on the research questions and hypotheses, a systematic review of the literature, and prior work in other low-risk cancers (see Supporting Materials). ${ }^{1,2,12,14-18}$ We used standard techniques to assess content validity, including review by design experts, content experts, and experts in risk perception, and pilot testing in a selected cohort of patients at the University of Michigan.

\section{Patient Perceptions Regarding Disease Recurrence and Mortality Risk From Thyroid Cancer}

Patients were asked to "Imagine 100 patients with the same size and type of thyroid cancer as you" followed by "How many of these patients do you think will have their thyroid cancer come back within the next 10 years (that is have cancer recurrence)?" and "How many of these patients do you think will die in the next 10 years?" Response categories for both questions were based on an 11-point scale with increments of 10 and endpoints of $\leq 5$ and $\geq 95$. For the analysis, we converted responses into percentages (11-point scale: increments of $10 \%$ and endpoints of $\leq 5 \%$ and $\geq 95 \%$ ). Using categories similar to those in breast cancer studies conducted by members of our team to assess risk estimation, we categorized the outcomes into 2 groups: reasonably accurate estimate (risk perception of $\leq 5 \%$ or $10 \%$ ) versus overestimation (risk perception of $20 \%$ to $\geq 95 \%$ ) of disease recurrence and mortality risk in this analytic sample of patients with 


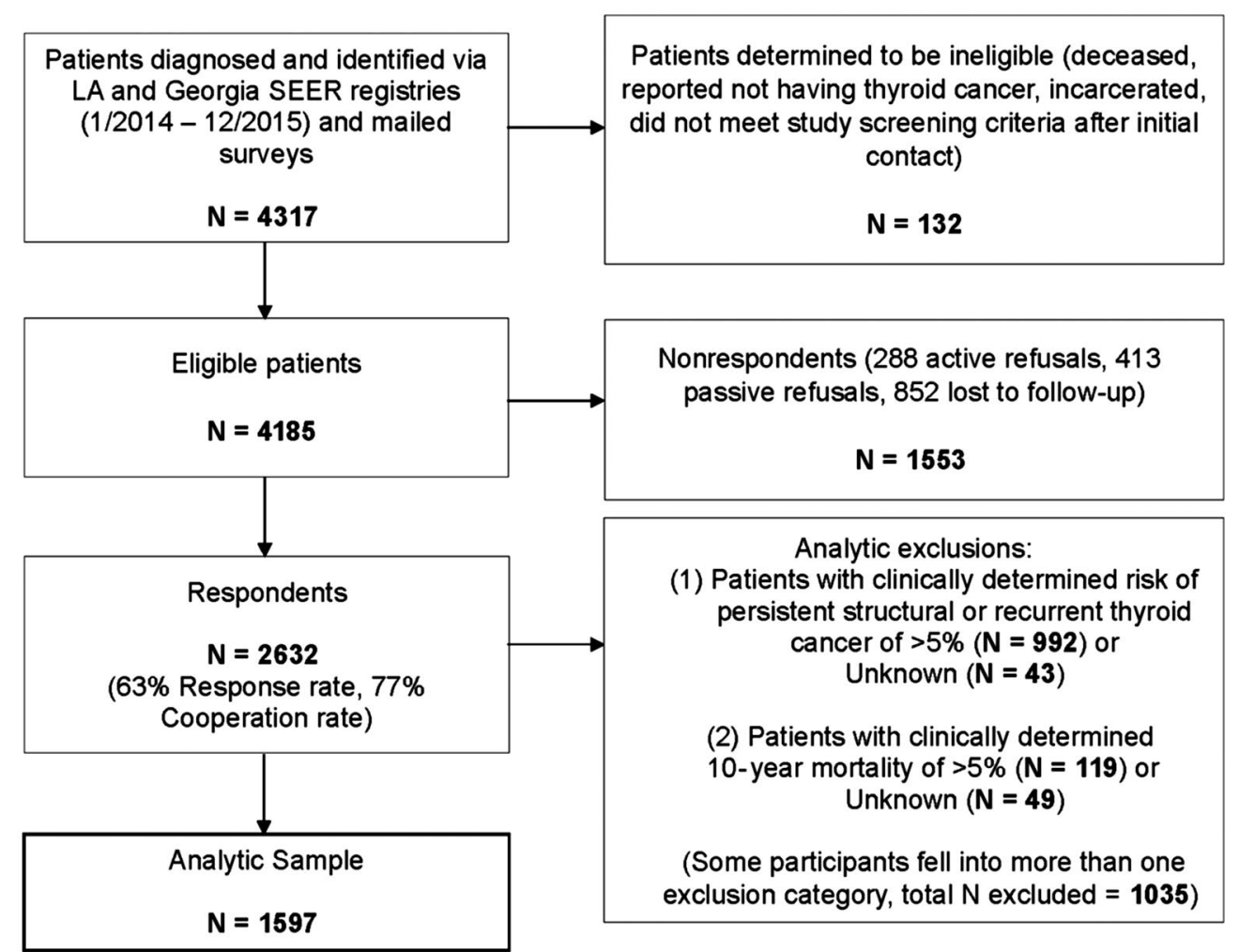

Figure 1. Flow diagram demonstrating analytic sample selection. The response rate was $63 \%$ and the cooperation rate was $77 \%$. LA indicates Los Angeles County; SEER, Surveillance, Epidemiology, and End Results program.

clinically determined 10 -year recurrence and mortality risks of $\leq 5 \%$. ${ }^{14,19}$

\section{Patient Report of Worry}

To determine worry regarding disease recurrence and death from thyroid cancer, we adapted questions from worry scales used in the breast cancer literature. ${ }^{15-17}$ Patients were asked to rate how much they worried about "thyroid cancer coming back" and "death from thyroid cancer" within the past month. A 5-point Likert scale with the following response categories was used: 1) not at all; 2) a little; 3) somewhat; 4) quite a bit; and 5) very much. For the analysis, we defined patient-reported "worry" as those who worried somewhat, quite a bit, or very much. This categorization was used to identify those patients who had a degree of worry that potentially could influence health behaviors.

\section{Patient Report of QOL}

The Patient-Reported Outcomes Measurement Information System (PROMIS) Global Health questionnaire (version 1.2), which is based on item response theory, was used to measure QOL. ${ }^{18}$ Physical and mental health raw scores were summed and converted into
T scores, which were normalized to a sample population that represented the 2000 general US population with a mean of 50 and a standard deviation of 10 . Accurate conversion to $\mathrm{T}$ scores requires the completion of all 4 items for each subscale, and therefore we excluded patients who did not respond to all the required items (38 patients for physical QOL and 38 patients for mental QOL). Higher scores indicated better outcomes such that higher physical QOL scores indicated better physical function.

\section{Covariates}

We obtained the following demographic characteristics from the survey: sex (female vs male), race/ethnicity (Hispanic, black, white, or other), and highest level of education attained ( $\leq$ high school diploma, some college, and $\geq$ college degree). With regard to race/ethnicity, patients were asked to check all options that best described their race/ethnicity. Similar to prior studies, patients who selected multiple options for race/ethnicity were assigned to 1 category according to the following priority order: Hispanic, black, white, and other. ${ }^{5,20}$ Each individual was analyzed as having 1 race/ethnicity. Clinical cancer characteristics obtained from the SEER registries were age 
at the time of diagnosis (in years), ICD-O-3 morphology codes for thyroid cancer diagnosis, and the tumor size and extension from both the clinical and pathologic sources that the SEER registries used to derive a $T$ classification following definitions from the American Joint Committee on Cancer seventh edition staging system. ${ }^{21}$

\section{Statistical Analyses}

Descriptive statistics were generated for all categorical variables, and we have reported nonweighted frequencies and weighted percentages. We used multivariable logistic regression analysis to determine factors associated with the overestimation of recurrence risk and of mortality risk. We reported adjusted odds ratios (ORs) with 95\% CIs for all logistic regression models, with $P$ values $<.05$ considered to be statistically significant.

Rao-Scott adjusted chi-square tests were used to test for a relationship between patients' perception of risk of disease recurrence and mortality risk (reasonably accurate estimate or overestimate) and worry about recurrence and death, respectively.

We compared between-group mean physical and mental health $\mathrm{T}$ scores of the analytic sample, grouped by patients' perceived risk (reasonably accurate estimate or overestimate) of recurrence and of mortality, using a regression analysis to the general US population. A meaningful change in global physical or mental health was defined as a minimal difference of approximately one-half of an SD, or 5 points, compared with the mean for the sample population that represented the 2000 general US Census (mean T score, 50). ${ }^{18}$

All statistical analyses incorporated weights to account for differential sampling and to reduce potential nonresponse bias. This included the use of design weights to account for differential probability of sample selection and nonresponse weights to account for disproportionate nonresponse rates across different patient subgroups. This weighting aims to generate statistical inferences that are more representative of the target population. ${ }^{22,23}$ The percentages and ORs reported were weighted and the number of participants, when provided, was unweighted for clarity. Analyses were performed using STATA (version 15.1; StataCorp LLC, College Station, Texas), R (version 3.5.2; R Foundation for Statistical Computing, Vienna, Austria), and SAS (version 9.4; SAS Institute Inc, Cary, North Carolina) statistical software.

\section{RESULTS}

Table 1 provides the distribution of patient demographics, tumor characteristics, and risk perception of the study
TABLE 1. Distribution of Patient Demographics, Tumor Characteristics, and Risk Perception of the Analytic Sample $(N=1597)$

\begin{tabular}{lr}
\hline Characteristic & No. $^{\mathrm{a}}(\%)^{\mathrm{b}}$ \\
\hline Sex & \\
$\quad$ Female & $1285(80.9)$ \\
$\quad$ Male & $312(19.1)$ \\
Age at diagnosis, y & \\
$\quad \leq 44$ & $517(35.3)$ \\
$45-54$ & $386(23.4)$ \\
$55-64$ & $405(24.1)$ \\
$\geq 65$ & $289(17.2)$ \\
Race/ethnicity & \\
White & $997(58.5)$ \\
Black & $200(14.3)$ \\
Hispanic & $248(17.2)$ \\
Other & $138(10.0)$ \\
Highest level of education & \\
$\quad \leq$ High school diploma & $362(23.0)$ \\
Some college & $488(31.1)$ \\
$\geq$ College degree & $719(45.9)$ \\
Derived T classification & \\
$\quad$ T1a & $820(52.5)$ \\
T1b & $438(26.7)$ \\
T2 & $339(20.8)$ \\
Risk perception & \\
Overestimate 10-y recurrence risk & $354(24.7)$ \\
Overestimate 10-y mortality risk & $178(12.5)$ \\
\hline aUnweighted number. & \\
${ }^{b}$ Weighted percentage. & \\
\end{tabular}

sample. Of the 1597 low-risk patients in the sample, 1285 (80.9\%) were female, 997 (58.5\%) were white, and 719 $(45.9 \%)$ had at least a college degree. The majority had T1a $(52.5 \%)$ or $\mathrm{T} 1 \mathrm{~b}(26.7 \%)$ disease at the time of diagnosis. Approximately one-quarter of the sample (24.7\%) overestimated their 10-year recurrence risk from thyroid cancer, and $12.5 \%$ overestimated their 10 -year mortality risk.

Figures 2 and 3 show the multivariable adjusted ORs and 95\% CIs of correlates of overestimation of disease recurrence and mortality risk, respectively. A lower educational level was associated with the overestimation of both risk of disease recurrence ( $\leq$ high school diploma: OR, 1.64 [95\% CI, 1.16-2.31]; and some college education: OR, 1.36 [95\% CI, 1.02-1.81]) and mortality risk ( $\leq$ high school diploma: OR, 1.86; 95\% CI, 1.182.93) compared with having $\geq$ college degree. The odds of overestimating recurrence risk were found to be greater among Hispanic patients (OR, 1.44; 95\% CI, 1.02-2.03) compared with their white counterparts. Sensitivity analysis excluding patients who self-reported persistent or recurrent thyroid cancer or an unknown disease status on the patient survey (new total analytic cohort of 1422 patients) yielded similar results, with a lower educational level associated with the overestimation of both risk of recurrence ( $\leq$ high school diploma: OR, 1.60 [95\% CI, 1.10-2.34]; and some college education: OR, 1.57 


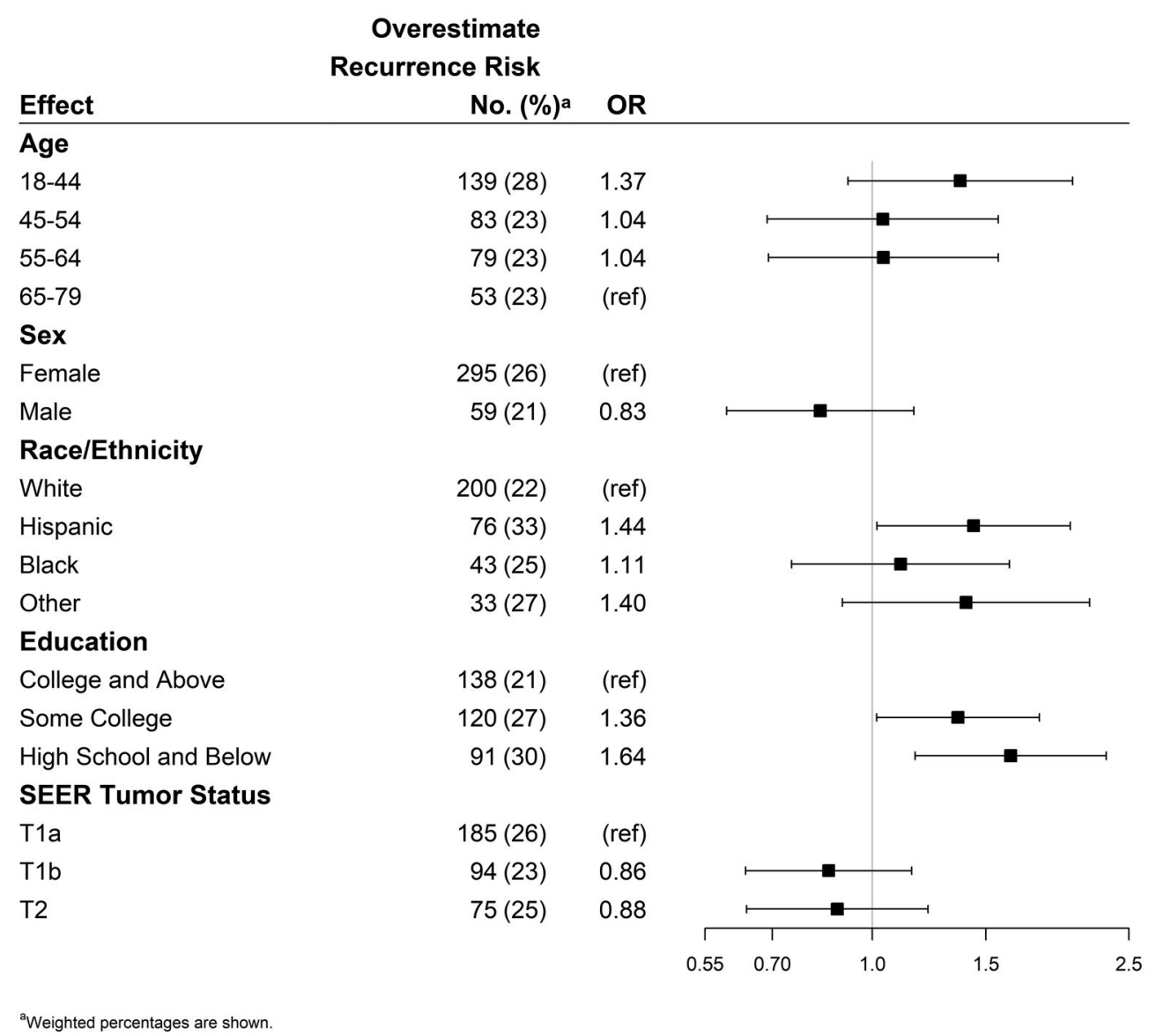

Figure 2. Multivariable adjusted odds ratios (ORs) and $95 \% \mathrm{Cls}$ of characteristics associated with overestimation of recurrence risk. SEER indicates Surveillance, Epidemiology, and End Results program.

[95\% CI, 1.15-2.14]) and mortality risk ( $\leq$ high school diploma: OR, 1.74; 95\% CI, 1.04-2.91) compared with having $\geq$ college degree. Similarly, when we restricted the cohort to patients who had undergone both a total thyroidectomy and radioactive iodine ablation (new total analytic cohort of 585 patients), a lower educational level again was found to be associated with overestimating recurrence risk ( $\leq$ high school diploma: OR, $2.06[95 \%$ CI, 1.19-3.57]; and some college education: OR, 1.67 [95\% CI, 1.03-2.70]) and mortality risk ( $\leq$ high school diploma: OR, 2.49 [95\% CI, 1.15-5.42]; and some college education: OR, 2.07 [95\% CI, 1.05-4.04]) compared with having $\geq$ college degree. However, in both sensitivity analyses, Hispanic ethnicity was no longer found to be associated with overestimation of recurrence risk.

Figure 4 shows the distribution of worry regarding disease recurrence and death from thyroid cancer relative to patient perception of recurrence and mortality risks. Worry about recurrence $(62.0 \%$ vs $30.8 \% ; P<.001)$ and death $(43.0 \%$ vs $19.0 \% ; P<.001)$ was greater among those who overestimated their risks of recurrence and mortality, respectively, compared with those who had a reasonably accurate risk estimate. Sensitivity analysis with patient-reported "worry" defined as any worry yielded similar results, with greater worry regarding disease recurrence $(79.5 \%$ vs $55.5 \% ; P<.001)$ and death $(59.6 \%$ vs $36.8 \% ; P<.001)$ noted among those who overestimated their risk of recurrence and mortality risk, respectively, compared with those who had a reasonably accurate risk estimate. Similarly, when we excluded patients who self-reported a history of depression (new total analytic cohort of 1275 patients), there still was greater worry regarding recurrence $(62.4 \%$ vs $29.3 \% ; P<.001)$ and death $(44.6 \%$ vs $18.1 \% ; P<.001)$ noted among patients who overestimated their risk of recurrence and mortality risk, respectively, compared with patients with reasonably accurate risk estimates.

Figure 5 illustrates physical and mental QOL by patient perception of recurrence and mortality risks. Compared with the 2000 general US population, a lower 


\section{Overestimate}

Mortality Risk

\section{Effect}

\section{Age}

$18-44$

45-54

55-64

65-79

Sex

Female

Male

\section{Race/Ethnicity}

White

Hispanic

Black

Other

Education

College and Above

Some College

High School and Below

SEER Tumor Status

T1a

$\mathrm{T} 1 \mathrm{~b}$

T2
No. $(\%)^{a}$ OR

$\begin{array}{ll}58(12) & 0.92 \\ 35(10) & 0.71 \\ 50(14) & 1.03 \\ 35(15) & \text { (ref) }\end{array}$

$135(12)$

43 (15)

(ref)

1.28

98 (11)

(ref)

38 (17) $\quad 1.49$

27 (15) $\quad 1.53$

14 (11) $\quad 1.23$

$\begin{array}{ll}62(10) & \text { (ref) } \\ 58(13) & 1.38 \\ 52(17) & 1.86 \\ & \\ 91(13) & \text { (ref) } \\ 51(13) & 1.01 \\ 36(12) & 0.87\end{array}$
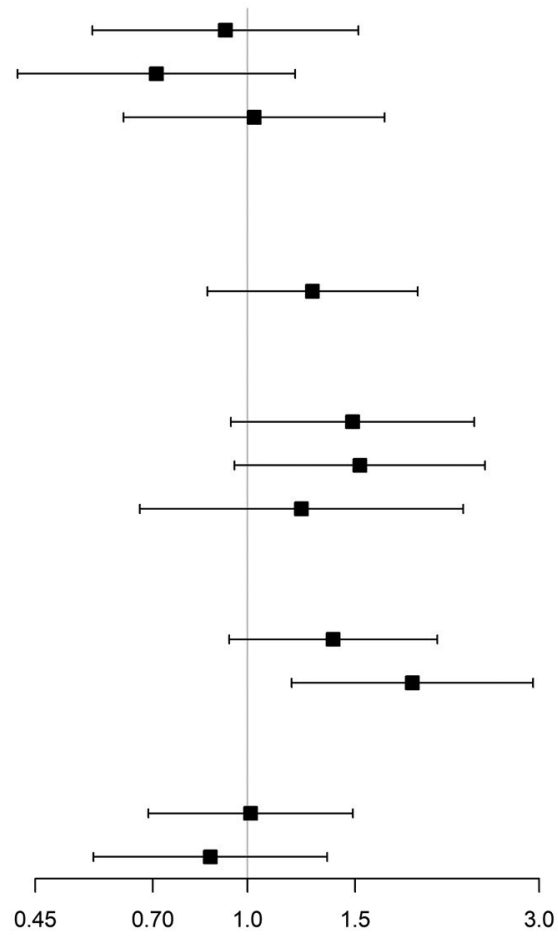

${ }^{a}$ Weighted percentages are shown.

Figure 3. Multivariable adjusted odds ratios (ORs) and $95 \% \mathrm{Cls}$ of characteristics associated with overestimation of mortality risk. SEER indicates Surveillance, Epidemiology, and End Results program.

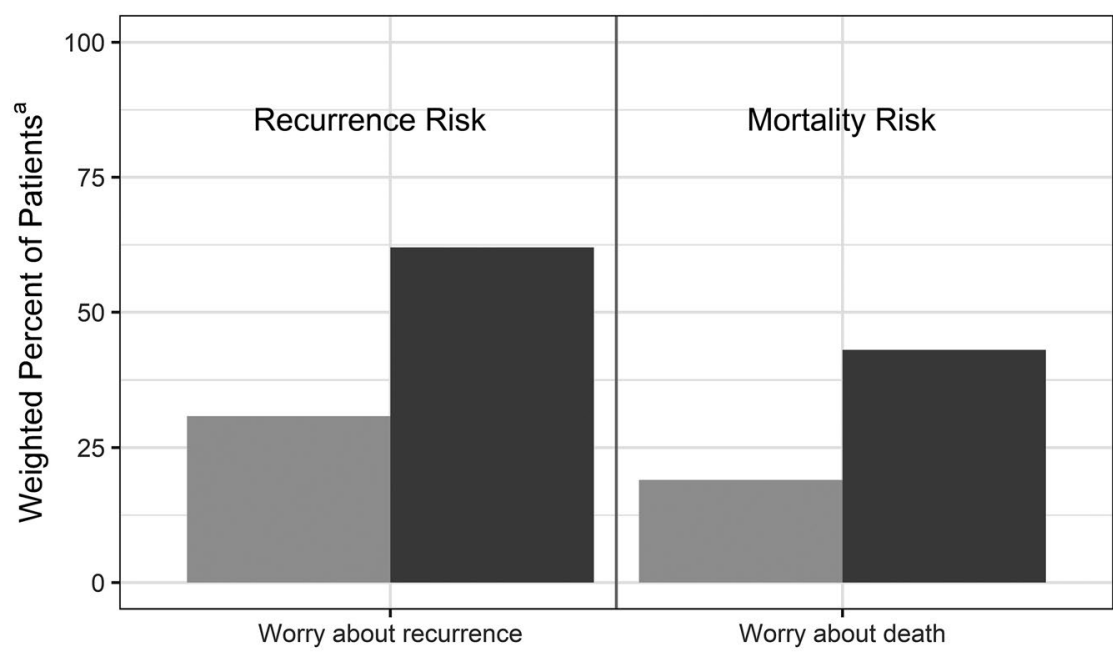

Worry in relation to risk

Reasonably accurate estimate

Overestimate

${ }^{a}$ Weighted percentages are shown.

Figure 4. Patient-reported worry regarding disease recurrence and death from thyroid cancer relative to patient perception of recurrence and mortality risks, respectively. Rao-Scott adjusted chi-square $P$ values were $<.001$. 


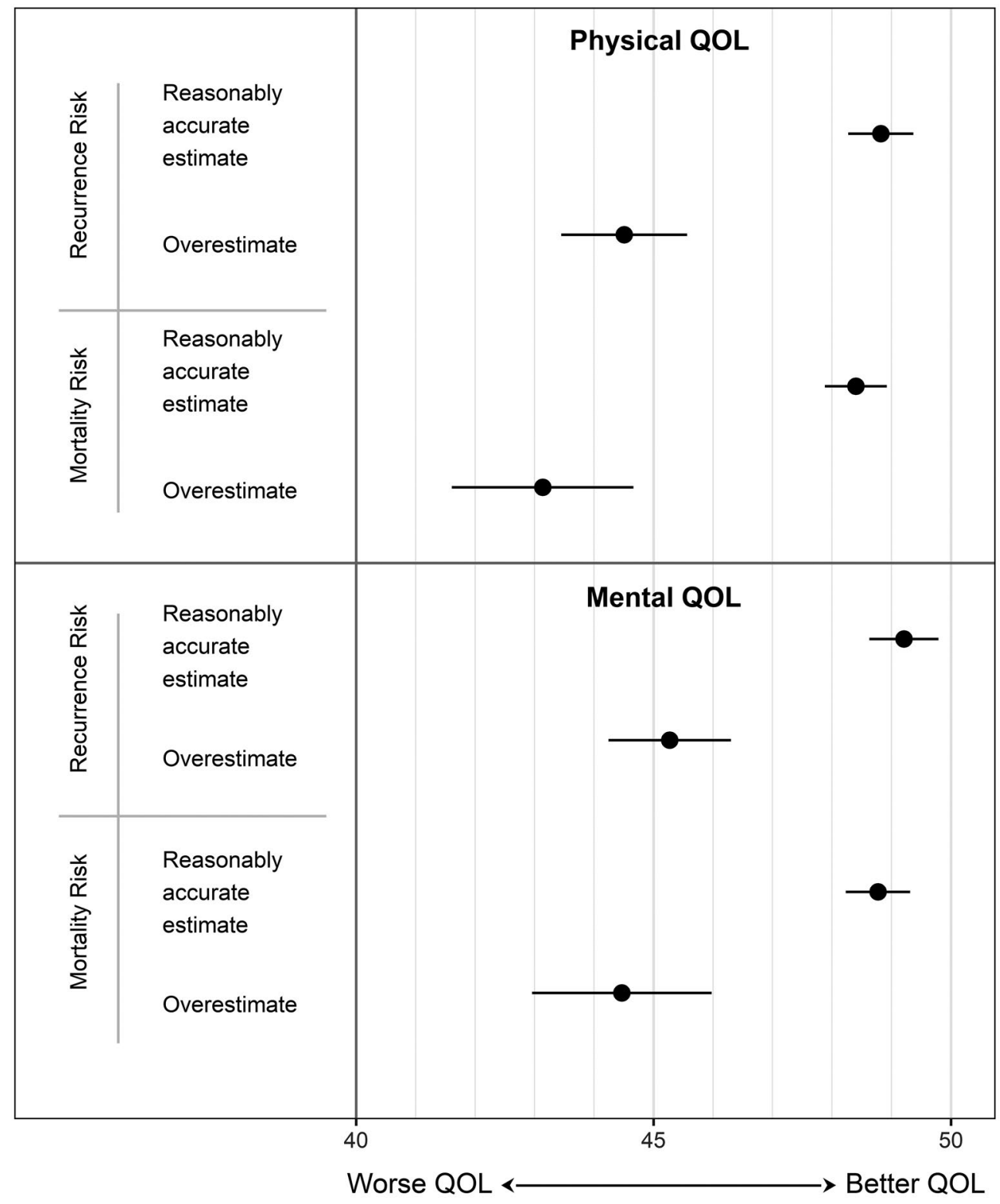

Mean T-score $(95 \% \mathrm{Cl})$

Figure 5. Physical and mental quality of life (QOL) by patient perception of disease recurrence and mortality risks. Lower $T$ scores indicated a worse QOL. The mean T scores and 95\% Cls were compared with those for the 2000 general US population. A meaningful change was defined as a minimal difference of 5 points compared with the mean $T$ score of 50 for the general population.

mean physical QOL T score among patients who overestimated their mortality risk met the criteria for meaningful change (mean T score, 43.1; 95\% CI, 41.6-44.7). Mean physical and mental QOL T scores in patients who overestimated their recurrence risk or who had reasonably accurate risk estimates did not meet the criteria for a meaningful change compared with the general US population. Furthermore, when compared with patients with reasonably accurate risk estimates, those who overestimated their recurrence risk were more likely to have low physical $(36.9 \%$ with at least 1 SD below the mean vs $19.3 \% ; P<.001)$ and mental $(28.1 \%$ with at least 1 SD below the mean vs $17.3 \% ; P<.001)$ QOL T scores. Similarly, those who overestimated mortality risk also were more likely to have low physical $(43.6 \%$ with at least 1 SD below the mean vs $21.0 \% ; P<.001)$ and mental 
(31.5\% with at least 1 SD below the mean vs $18.2 \%$; $P<.001)$ QOL T scores. In sensitivity analysis excluding patients who self-reported a history of depression (new total analytic cohort of 1275 patients), the mean physical QOL T score in patients who overestimated their mortality risk was 44.9 (95\% CI, 43.2-46.6). Because a T score of 45.0 is within the $95 \% \mathrm{CI}$, this was no longer statistically significantly different from that of the general US population.

\section{DISCUSSION}

The current large, population-based study has provided novel insights regarding risk perception among patients with low-risk thyroid cancer. Survivors who were less educated and those of Hispanic ethnicity were more likely to overestimate their recurrence risk, with the former also more likely to overestimate their mortality risk. These inaccurate risk perceptions were associated with increased worry regarding disease recurrence and death, respectively, and decreased physical QOL among those who overestimated their mortality risk.

It is plausible that the association between lower educational level and overestimation of recurrence and mortality risks is secondary to lower health literacy and numeracy. In a study of women attending a high-risk family history clinic, Rutherford et al demonstrated a correlation between the educational level attained and health literacy, and a correlation between low health literacy and an inaccurate risk perception of developing breast cancer. ${ }^{24}$ Moreover, Lipkus et al demonstrated that breast cancer patients with greater numeracy were more likely to provide estimates of cancer-free survival that matched estimates provided by a decision aid program for different treatment options compared with patients with lower numeracy. ${ }^{25}$

The greater likelihood of overestimating recurrence risk among Hispanic patients may be due in part to physician-patient communication barriers or differences in acculturation, 2 topics that to the best of our knowledge remain largely unexplored in patients with thyroid cancer. Alternatively, it may be related to differential access to and quality of thyroid cancer care for Hispanic patients compared with their white counterparts, with the potential for true differences in health outcomes. ${ }^{26-28}$

Worry regarding disease recurrence and death is common among patients with thyroid cancer. ${ }^{5,6}$ However, to the best of our knowledge, there is a lack of data regarding the relationship between risk estimation and worry in survivors of thyroid cancer. Consistent with studies in the breast cancer literature, we found that patients who overestimated their risks of recurrence and mortality were more likely to report cancer-related worry. ${ }^{19,29,30}$ In addition, Papaleontiou et al demonstrated that in a cohort of patients with DTC, a lower educational level was associated with more worry about recurrence and death from thyroid cancer, and Hispanic patients were more worried about death compared with their white counterparts 2 to 4 years after diagnosis. ${ }^{5}$ Despite the observed crosssectional association between recurrence and mortality risk overestimation and cancer-related worry, with both more likely to occur among less educated and/or Hispanic patients, to our knowledge the causality between these 2 variables remains unknown. Risk overestimation leading to increased cancer-related worry is as conceivable as is increased worry leading patients to report higher risk estimates. Further research is needed to elucidate the relationship between risk estimates and worry.

The findings of the current study have provided new insights into the relationship between thyroid cancer survivorship and QOL. Although QOL is an important area of research, prior studies have been discordant regarding the association between thyroid cancer history and QOL. ${ }^{6-8}$ In our analytic sample, only patients who overestimated their mortality risk demonstrated a meaningful decrease in physical QOL compared with the general population, although the decrease was no longer statistically significantly different from that of the general US population when we excluded patients who selfreported a history of depression. This suggests that a better understanding of risk perception is an important aspect of helping to understand QOL among survivors of thyroid cancer.

A major strength of the current study was the combination of clinical cancer data from the SEER registries and a complementary patient survey, which provided granular details regarding patient report of risk estimation, worry, and QOL. Collaboration with the Georgia and Los Angeles County SEER sites provided for a large and diverse population-based cohort of low-risk thyroid cancer survivors with adequate representation of Hispanic and black patients. Additional strengths of the current study included a high response rate among surveyed patients, a focus on an understudied cancer, and the use of the validated PROMIS scale to assess physical and mental QOL. The multiple sensitivity analyses performed were another strength of the study, although the new cohorts were smaller and therefore it is unclear whether some of the subsequent results were no longer statistically significant due to the exclusion of a specific variable or due to a smaller cohort size. 
Some potential limitations should be noted. First, because the current study was cross-sectional, the observed associations do not suggest causation. Second, although mortality data were straightforward, patient-reported risk of thyroid "cancer recurrence" was compared with data regarding clinically determined risk of persistent structural or recurrent thyroid cancer. We did not distinguish biochemical versus structural recurrent or persistent disease in the questionnaire, largely because although patients likely will recall being told their cancer is cured, recurred, or still present, they may not be able to differentiate biochemical versus structural recurrence. However, we did add sensitivity analyses to assess the translatability of findings in more specific cohorts. Third, the cohort included only patients from 2 geographic areas and therefore may not be representative of all patients with thyroid cancer. However, the population covered by SEER is comparable to the general US population. Fourth, patients were not asked about resources they used to learn about thyroid cancer or their comfort in communicating with physicians, both of which may influence their risk perception. Furthermore, the survey did not assess health literacy and numeracy or evaluate acculturation among Hispanic patients, information that could provide insight into risk overestimation among patients who are less educated and Hispanic patients.

The study implications are relevant to patients and physicians. Despite the favorable prognosis of low-risk thyroid cancer, many vulnerable patients, including those with a lower educational level and Hispanic ethnicity, inaccurately perceived their risk of disease recurrence and/ or death, reported more worry, and experienced decreased physical QOL. Patient education that is appropriate for individuals of varying educational levels and culturally sensitive, and improved risk communication by physicians, are necessary to address inaccurate risk perceptions. Improving psychosocial support, including the availability of online websites and in-person support groups, for patients with thyroid cancer-related worry also is imperative. Effective communication and appropriate reassurance by physicians may help patients to create a framework for understanding their cancer prognosis so that they can make informed treatment decisions and adequately cope with the psychosocial stress related to having a cancer diagnosis. Such interventions may translate into an improved QOL for survivors of thyroid cancer.

\section{FUNDING SUPPORT}

Supported by National Cancer Institute (NCI) grant R01 CA201198 (Principal Investigator: Megan R. Haymart; Co-investigators: Ann S. Hamilton, Kevin C. Ward, Sarah T. Hawley, and Brian J. Zikmund-Fisher). The collection of cancer incidence data used in this study was supported by the California Department of Public Health pursuant to California Health and Safety Code Section 103885; the Centers for Disease Control and Prevention's (CDC's) National Program of Cancer Registries under cooperative agreement 5NU58DP003862-04/DP003862; and the NCI's Surveillance, Epidemiology, and End Results (SEER) Program under contract HHSN261201000035C awarded to the University of Southern California. The collection of cancer incidence data in Georgia was supported by contract HHSN261201800003I, task order HHSN26100001 from the NCI and cooperative agreement 5NU58DP003875-04 from the CDC. The ideas and opinions expressed herein are those of the authors, and endorsement by the State of California and State of Georgia Departments of Public Health, the NCI, and the CDC or their contractors and subcontractors is not intended nor should be inferred.

\section{CONFLICT OF INTEREST DISCLOSURES}

Debbie W. Chen receives funding from grant T32DK07245 from the National Institute of Diabetes and Digestive and Kidney Diseases. Megan R. Haymart receives funding from R01 HS024512 from the Agency for Healthcare Research and Quality. The other authors made no disclosures.

\section{AUTHOR CONTRIBUTIONS}

Debbie W. Chen: Conceptualization, visualization, writing-original draft, and writing-review and editing. David Reyes-Gastelum: Data curation, formal analysis, methodology, and writing-review and editing. Lauren P. Wallner: Visualization and writing-review and editing. Maria Papaleontiou: Visualization and writing-review and editing. Ann S. Hamilton: Conceptualization, data curation, funding acquisition, methodology, visualization, and writing-review and editing. Kevin C. Ward: Conceptualization, data curation, funding acquisition, methodology, visualization, and writing-review and editing. Sarah T. Hawley: Conceptualization, funding acquisition, methodology, visualization, and writing-review and editing. Brian J. Zikmund-Fisher: Conceptualization, funding acquisition, methodology, visualization, and writing-review and editing. Megan R. Haymart: Conceptualization, data curation, funding acquisition, methodology, visualization, writing-original draft, and writing-review and editing. All authors reviewed and approved the final version of the article.

\section{REFERENCES}

1. Haugen BR, Alexander EK, Bible KC, et al. 2015 American Thyroid Association Management Guidelines for Adult Patients with Thyroid Nodules and Differentiated Thyroid Cancer: The American Thyroid Association Guidelines Task Force on Thyroid Nodules and Differentiated Thyroid Cancer. Thyroid. 2016;26:1-133.

2. Banerjee M, Muenz DG, Chang JT, Papaleontiou M, Haymart MR. Tree-based model for thyroid cancer prognostication.J Clin Endocrinol Metab. 2014;99:3737-3745.

3. Tuttle RM, Haugen B, Perrier ND. Updated American Joint Committee on Cancer/Tumor-Node-Metastasis Staging System for Differentiated and Anaplastic Thyroid Cancer (Eighth Edition): what changed and why? Thyroid. 2017;27:751-756.

4. American Cancer Society. Thyroid cancer survival rates, by type and stage. Accessed February 20, 2017. https://www.cancer.org/cancer/ thyroid-cancer/detection-diagnosis-staging/survival-rates.html

5. Papaleontiou M, Reyes-Gastelum D, Gay BL, et al. Worry in thyroid cancer survivors with a favorable prognosis. Thyroid. 2019; 29:1080-1088.

6. Hedman C, Djarv T, Strang P, Lundgren CI. Determinants of long-term quality of life in patients with differentiated thyroid carcinoma-a population-based cohort study in Sweden. Acta Oncol. 2016;55:365-369.

7. Schultz PN, Beck ML, Stava C, Vassilopoulou-Sellin R. Health profiles in 5836 long-term cancer survivors. Int J Cancer. 2003;104:488-495.

8. Hoftijzer HC, Heemstra KA, Corssmit EP, van der Klaauw AA, Romijn JA, Smit JW. Quality of life in cured patients with differentiated thyroid carcinoma. J Clin Endocrinol Metab. 2008;93:200-203.

9. Malterling RR, Andersson RE, Falkmer S, Falkmer U, Nilehn E, Jarhult J. Differentiated thyroid cancer in a Swedish county-long-term results and quality of life. Acta Oncol. 2010;49:454-459. 
10. Dillman DA, Smyth JD, Christian LM. Internet, Mail, and MixedMode Surveys: The Tailored Design Method. 3rd ed. Wiley; 2009.

11. American Association for Public Opinion Research. Response rates-an overview. Accessed February 20, 2017. https://www.aapor.org/Educa tion-Resources/For-Researchers/Poll-Survey-FAQ/Response-Rates-AnOverview.aspx

12. Tuttle RM, Tala H, Shah J, et al. Estimating risk of recurrence in differentiated thyroid cancer after total thyroidectomy and radioactive iodine remnant ablation: using response to therapy variables to modify the initial risk estimates predicted by the new American Thyroid Association staging system. Thyroid. 2010;20:1341-1349.

13. Young JL Jr, Roffers SD, Ries LAG, Fritz AG, Hurlbut AA. SEER Summary Staging Manual-2000: Codes and Coding Instructions. National Cancer Institute; 2001. NIH publication 01-4969.

14. Lee KL, Janz NK, Zikmund-Fisher BJ, et al. What factors influence women's perceptions of their systemic recurrence risk after breast cancer treatment? Med Decis Making. 2018;38:95-106.

15. Janz NK, Hawley ST, Mujahid MS, et al. Correlates of worry about recurrence in a multiethnic population-based sample of women with breast cancer. Cancer. 2011;117:1827-1836.

16. Janz NK, Friese CR, Li Y, Graff JJ, Hamilton AS, Hawley ST. Emotional well-being years post-treatment for breast cancer: prospective, multi-ethnic, and population-based analysis. J Cancer Surviv. 2014; 8:131-142.

17. Janz NK, Li Y, Beesley LJ, et al. Worry about recurrence in a multi-ethnic population of breast cancer survivors and their partners. Support Care Cancer. 2016;24:4669-4678.

18. HealthMeasures. Patient-Reported Outcomes Measurement Information System. Accessed February 20, 2017. http://www.healt hmeasures.net/explore-measurement-systems/promis

19. Hawley ST, Janz NK, Griffith KA, et al. Recurrence risk perception and quality of life following treatment of breast cancer. Breast Cancer Res Treat. 2017; 161:557-565.
20. Jagsi R, Pottow JA, Griffith KA, et al. Long-term financial burden of breast cancer: experiences of a diverse cohort of survivors identified through population-based registries. J Clin Oncol. 2014;32: 1269-1276.

21. Adamo M, Dickie L, Ruhl J. 2014 SEER Program Coding and Staging Manual. National Cancer Institute; 2014.

22. Groves RM, Fowler FJ, Couper MP, Lepkowski JM, Singer E, Tourangean R. Survey Methodology. 2nd ed. Wiley; 2009.

23. Kish L. Survey Sampling. John Wiley \& Sons Inc; 1965.

24. Rutherford EJ, Kelly J, Lehane EA, et al. Health literacy and the perception of risk in a breast cancer family history clinic. Surgeon. 2018;16:82-88.

25. Lipkus IM, Peters E, Kimmick G, Liotcheva V, Marcom P. Breast cancer patients' treatment expectations after exposure to the decision aid program Adjuvant Online: the influence of numeracy. Med Decis Making. 2010;30:464-473.

26. Sosa JA, Bowman HM, Tielsch JM, Powe NR, Gordon TA, Udelsman R. The importance of surgeon experience for clinical and economic outcomes from thyroidectomy. Ann Surg. 1998;228: 320-330.

27. Sosa JA, Mehta PJ, Wang TS, Yeo HL, Roman SA. Racial disparities in clinical and economic outcomes from thyroidectomy. Ann Surg. 2007;246:1083-1091.

28. Shah SA, Adam MA, Thomas SM, et al. Racial disparities in differentiated thyroid cancer: have we bridged the gap? Thyroid. 2017; 27:762-772.

29. Kelly KM, Ajmera M, Bhattacharjee S, et al. Perception of cancer recurrence risk: more information is better. Patient Educ Couns. 2013;90:361-366.

30. Rondanina G, Puntoni M, Guerrieri-Gonzaga A, Marra D, Bonanni B, DeCensi A. Worry and risk perception of breast cancer in a prevention trial of low dose tamoxifen in midlife postmenopausal hormone users. Breast. 2017;34:108-114. 